\title{
Immature platelet fraction based diagnostic predictive scoring model for immune thrombocytopenia
}

\author{
Min Ji Jeon ${ }^{1}$, Eun Sang Yu ${ }^{1}$ Ka-Won Kang², Byung-Hyun Lee ${ }^{2}$, Yong Park², Se Ryeon Lee ${ }^{3}$, Hwa Jung Sung ${ }^{3}$, \\ Soo Yong Yoon ${ }^{4}$, Chul Won Choi ${ }^{1}$, Byung Soo Kim ${ }^{2}$, and Dae Sik Kim ${ }^{1}$
}

${ }^{1}$ Division of Hematology-Oncology, Department of Internal Medicine, Korea University Guro Hospital, Seoul; ${ }^{2}$ Division of HematologyOncology, Department of Internal Medicine, Korea University Anam Hospital, Seoul; ${ }^{3}$ Division of Hematology-Oncology, Department of Internal Medicine, Korea University Ansan Hospital, Ansan; ${ }^{4}$ Department of Laboratory Medicine, Korea University Guro Hospital, Seoul, Korea

Received: March 18, 2019

Revised : March 24, 2019

Accepted: March 24, 2019

\section{Correspondence to}

Dae Sik Kim, M.D.

Division of Hematology-

Oncology, Department of

Internal Medicine, Korea

University Guro Hospital, 148

Gurodong-ro, Guro-gu, Seoul

०8308, Korea

Tel: +82-2-2626-3062

Fax: +82-2-2626-2208

E-mail: kay9801@naver.com https://orcid.org/0000-0001$8424-8561$
Background/Aims: The diagnosis of immune thrombocytopenia (ITP) is based on clinical manifestations and there is no gold standard. Thus, even hematologic malignancy is sometimes misdiagnosed as ITP and adequate treatment is delayed. Therefore, novel diagnostic parameters are needed to distinguish ITP from other causes of thrombocytopenia. Immature platelet fraction (IPF) has been proposed as one of new parameters. In this study, we assessed the usefulness of IPF and developed a diagnostic predictive scoring model for ITP.

Methods: We retrospectively studied 568 patients with thrombocytopenia. Blood samples were collected and IPF quantified using a fully-automated hematology analyzer. We also estimated other variables that could affect thrombocytopenia by logistic regression analysis.

Results: The median IPF was significantly higher in the ITP group than in the non-ITP group (8.7\% vs. 5.1\%). The optimal cut-off value of IPF for differentiating ITP was $7.0 \%$. We evaluated other laboratory variables via logistic regression analysis. IPF, hemoglobin, lactate dehydrogenase (LDH), and ferritin were statistically significant and comprised a diagnostic predictive scoring model. Our model gave points to each of variables: 1 to high hemoglobin (>12 g/dL), low ferritin ( $\leq 177 \mathrm{ng} /$ $\mathrm{mL}$ ), normal LDH ( $\leq$ upper limit of normal) and IPF $\geq 7$ and $<10,2$ to IPF $\geq 10$. The final score was obtained by summing the points. We defined that ITP could be predicted in patients with more than 3 points.

Conclusions: IPF could be a useful parameter to distinguish IT'P from other causes of thrombocytopenia. We developed the predictive scoring model. This model could predict ITP with high probability.

Keywords: Thrombocytopenia; Immature platelet fraction; Immune thrombocytopenia

\section{INTRODUCTION}

Immune thrombocytopenia (ITP) is an immune-mediated acquired disease characterized by isolated thrombocytopenia [1]. ITP is usually considered a benign disease with no excess mortality compared to the general population. However, some patients did not respond to numerous treatments and suffered from a fatal hemorrhage or infection related to immunosuppressive therapy [2]. Although the exact mechanism of thrombocytopenia is unclear, it is now known to be associated with increased platelet destruction by a complex immune-mediated process [3]. ITP can be diagnosed based on history, physical examination, laboratory variables, 
and other causes of thrombocytopenia should be excluded. Patients with ITP show unremarkable peripheral blood smear morphology and physical examination except various bleeding manifestations [4]. Since there are no other definite laboratory or clinical parameters for the diagnosis of ITP [1], even aplastic anemia or hematologic malignancy is sometimes misdiagnosed as ITP and adequate treatment is delayed. Therefore, physicians are forced to consider bone marrow examination in a significant number of patients with severe thrombocytopenia in practice, unlike previous studies which assert that a routine bone marrow examination is not necessary for diagnosis $[5,6]$. Since both physicians and patients are reluctant to perform an invasive and painful bone marrow examination, other parameters such as immature platelet fraction (IPF) have been proposed to differentiate thrombocytopenia instead of bone marrow examination.

Reticulated platelets have been studied as a parameter in the evaluation of thrombocytopenia for decades. Reticulated platelets are the immature, youngest platelets released into the circulation by regenerated marrow megakaryocytes and reflect the rate of platelet turnover similar to reticulocytes of red blood cells [7-9]. Reticulated platelets contain a greater amount of RNA [10], and can be measured by flow cytometric analysis using the fluorescent dye which binds to their RNA [7]. Several researchers have improved flow cytometric methods; however, flow cytometry still has various limitations such as being time consuming, difficult sample preparation, requiring a skilled operator, high costs, and lack of quality control [11]. Therefore, Watanabe et al. [12] described a fully automated measurement of reticulated platelets, and several studies showed that the automated measurement of immature platelets using the hematology analyzer XE-2100 (Sysmex, Kobe, Japan) is clinically applicable [13-20].

Because a high IPF value is associated with increased platelet production [15], we can presume that ITP can be differentiated from thrombocytopenia due to marrow failure by using IPF. Some studies have shown that IPF could be used to make a differential diagnosis between ITP and other diseases causing thrombocytopenia (nonITP thrombocytopenia), such as bone marrow diseases, liver diseases and bone marrow suppression after chemotherapy. Briggs et al. [15] confirmed reproducibili- ty and stability of IPF using the Sysmex XE-2100, and suggested IPF as a parameter of bone marrow megakaryocyte activity. Abe et al. [13] and Pons et al. [20] also concluded IPF could be a useful marker to discriminate causes of thrombocytopenia. However, there have been only a few small-scale studies, and exact cut-off value, sensitivity and specificity of IPF have not yet been determined. In addition, since there are other causes of thrombocytopenia with increased platelet turnover, it is impossible to distinguish ITP patients exactly with IPF alone. Therefore, other parameters to complement IPF should be evaluated.

In this study, we compared IPF in patients with ITP and patients with thrombocytopenia of other causes to assess its usefulness as a differentiating parameter in thrombocytopenia. We also estimated other laboratory variables which can be combined with IPF to improve the diagnostic accuracy of ITP and developed a diagnostic predictive scoring model for IT'P.

\section{METHODS}

\section{Patients}

We retrospectively studied 568 patients with thrombocytopenia (platelet count $<100 \times 10^{9} / \mathrm{L}$ ) of various causes who presented to Korea University Guro Hospital from April 2013 to December 2017. We classified the patients into two groups: those diagnosed with ITP (ITP group) and those diagnosed with other causes of thrombocytopenia apart from ITP (non-ITP group). ITP was diagnosed on the basis of clinical manifestations and laboratory results, according to the International Working Group diagnostic criteria [1], but some patients underwent bone marrow examination for differential diagnosis. Causes of thrombocytopenia other than ITP included the following: primary bone marrow diseases like aplastic anemia, acute leukemia, multiple myeloma, and myelodysplastic syndrome, infection, drugs, disseminated intravascular coagulation, and liver diseases, etc. The Korea University Hospital Institutional Review Board approved the protocol of this study (2019GRoo72). Informed consent was waived by the board.

\section{Measurement of IPF and other variables}

We collected $3 \mathrm{~mL}$ of peripheral blood from each patient 
in K2-EDTA anticoagulant bottles (Becton Dickinson, Franklin Lakes, NJ, USA) on the day of thrombocytopenia confirmation. Samples were analyzed using the Sysmex XE-2100 to quantify IPF and routine complete blood count within 8 hours after collection. The Sysmex $\mathrm{XE}-2100$ is a fully-automated hematology analyzer using fluorescent dye and a semiconductor diode laser beam system. Two fluorescent dyes (polymethine and ozazine) penetrate into the cells, staining the RNA in the platelets. The stained cells are passed through a semiconductor diode laser beam and resulting forward scatter light (cell volume) and fluorescence intensity (RNA content) measured. The immature platelet fractions are distinguished by the intensity of their fluorescence because the youngest platelets contain a greater amount of RNA [15]. The IPF is expressed as a proportional value of the total optical platelet count. Several studies have demonstrated the stability and reproducibility of the IPF.

We also estimated other variables that could affect thrombocytopenia by logistic regression analysis, including hemoglobin, platelet count, white blood cell counts, reticulocyte count, protein, albumin, bilirubin, prothrombin, activated partial thromboplastin time, ferritin, lactate dehydrogenase (LDH), blood urea nitrogen, creatinine, and C-reactive protein.

\section{Statistical analysis}

All the data were statistically analyzed using SPSS version 20 (IBM Corp., Armonk, NY, USA). For continuous variables, the Student's $t$ test and the Mann-Whitney test were applied to compare statistical differences between the two groups. We used a chi-square test or Fisher's exact test for comparisons of categorical variables. We used receiver operating characteristic (ROC) curves to determine the cut-off value of IPF at the best sensitivity and specificity and the area under the curve was calculated. Logistic regression analysis with the laboratory variables was performed to access their diagnostic contribution. A $p$ values less than 0.05 were considered statistically significant.

\section{RESULTS}

\section{Patients' characteristics}

A total of 568 patients with thrombocytopenia (platelet count less than $100 \times 10^{9} / \mathrm{L}$ ) were evaluated in this study. Of these, 206 were diagnosed with ITP according to the definitions proposed by the International Working Group, and 362 were classified as having thrombocytopenia due to causes other than ITP. The most common cause of thrombocytopenia in the non-ITP group was primary bone marrow diseases $(n=132)$, followed by infection $(n=69)$, drugs $(n=56)$, liver disease $(n=40)$, disseminated intravascular coagulation $(\mathrm{n}=30)$, and others ( $\mathrm{n}=35)$ including chronic kidney disease, other autoimmune diseases, megaloblastic anemia, and bone marrow involvement of other cancers (Table 1).

\section{IPF and laboratory results}

The baseline laboratory data of the ITP group and nonITP group were compared in Table 2. The ITP group had a significantly higher hemoglobin concentration $(p<$ $0.001)$, white blood cell count $(p<0.001)$, and lower LDH $(p<0.001)$, ferritin level $(p<0.001)$. The median IPF (\%) was significantly higher in the ITP group; the median $\operatorname{IPF}(\%)$ (range) of the ITP group was $8.7 \%$ (1.3\% to $47.5 \%$ ) while that of the non-ITP group was 5.1\% (0.7\% to 31.5\%) $(p<0.001)$. In order to obtain the optimal cut-off value of IPF (\%) for the diagnosis of IT'P, ROC curve analysis was carried out. As a result, the optimal cut-off value of IPF (\%) for differentiating ITP was 7.0\% with a sensitivity of $61 \%$ and a specificity of $70 \%$ (Fig. 1A). Bone marrow examinations are not mandatory for the diagnosis of ITP, but some patients who have clinically diagnosed ITP without a bone marrow examination may be difficult to differentiate from those with early bone marrow disease. To exclude this possibility of misclassification, we performed subgroup analysis with the patients who had an accurate diagnosis with bone marrow examination. A total of 200 patients underwent bone marrow examination; 77 patients were classified into the ITP group and 123 patients into the non-ITP group. The median IPF (\%) was significantly higher in the ITP group. The median IPF (\%) (range) was $12.5 \%$ (1.9\% to $45.7 \%$ ) for the ITP group and $4.7 \%$ (0.7\% to $31.5 \%)$ for the non-ITP group. The optimal cut-off value was $7.8 \%$, with a sensitivity of $75 \%$ and a specificity of $76.7 \%$ (Fig. 1 B). The median IPF was higher in this subgroup and the sensitivity and specificity of the cut-off value were also higher than the former group. We confirmed that IPF could be a useful parameter for diagnosing ITP, but since IPF alone could 
Table 1. Baseline characteristics of patients included in the study

\begin{tabular}{|c|c|c|c|}
\hline \multirow{2}{*}{ Characteristic } & \multicolumn{3}{|c|}{ No. of patients } \\
\hline & Total & ITP & Non-ITP \\
\hline No. of patients & 568 & 206 & 362 \\
\hline Age, yr, median (range) & $57(15-92)$ & $53(15-92)$ & $61(16-91)$ \\
\hline \multicolumn{4}{|l|}{ Sex } \\
\hline Male & 263 & 78 & 185 \\
\hline Female & 303 & 126 & 177 \\
\hline \multicolumn{4}{|l|}{ Diagnosis } \\
\hline Bone marrow diseases ${ }^{\mathrm{a}}$ & & & 132 \\
\hline Infection & & & 69 \\
\hline Drug & & & 56 \\
\hline DIC & & & 30 \\
\hline Liver disease & & & 40 \\
\hline Others $^{\mathrm{b}}$ & & & 35 \\
\hline
\end{tabular}

ITP, immune thrombocytopenia; DIC, disseminated intravascular coagulopathy.

${ }^{a}$ Bone marrow diseases include aplastic anemia, acute leukemia, myelodysplastic syndrome, multiple myeloma, myelofibrosis, chronic lymphoid leukemia, hemophagocytic lymphohistiocytosis, and bone marrow invasion by lymphoma.

${ }^{\mathrm{b}}$ Others include chronic kidney disease, other autoimmune diseases, megaloblastic anemia, and bone marrow involvement of other cancers, and patients who are strongly suspected primary bone marrow disease without bone marrow examination due to their refusal.

Table 2. Baseline laboratory findings of patients

\begin{tabular}{|c|c|c|c|}
\hline \multirow{2}{*}{ Characteristic } & \multicolumn{2}{|c|}{ No. of patients } & \multirow{2}{*}{$p$ value } \\
\hline & $\operatorname{ITP}(n=206)$ & Non-ITP $(n=362)$ & \\
\hline \multicolumn{4}{|l|}{ IPF, \% } \\
\hline Total patients & $8.7(1.3-47.5)$ & $5.1(0.7-31.5)$ & $<0.001$ \\
\hline Confirmed with BM biopsy & $12.5(1.9-45 \cdot 7)$ & $4.7(0.7-31.5)$ & $<0.001$ \\
\hline $\mathrm{PLT}, \times 10^{9} / \mathrm{L}$ & $49(2-97)$ & $55(3-99)$ & 0.003 \\
\hline $\mathrm{Hb}, \mathrm{g} / \mathrm{dL}$ & $12.7(5.4-18.3)$ & $10.1(3 \cdot 3-16.1)$ & $<0.001$ \\
\hline WBC, $\times 10^{9} / \mathrm{L}$ & $5 \cdot 9(1.1-16.1)$ & $4.6(0.3-242.7)$ & $<0.001$ \\
\hline Reticulocyte, \% & $1.44(0.18-8.12)$ & $1.51(0-8.65)$ & 0.942 \\
\hline Protein, g/dL & $7.2(4.7-8.4)$ & $6.7(3.5-63)$ & $<0.001$ \\
\hline Albumin, g/dL & $4.1(2.4-4.8)$ & $3.6(1.8-32)$ & $<0.001$ \\
\hline Bilirubin, mg/dL & $0.5(0.2-3.9)$ & $0.7(0.1-15.4)$ & $<0.001$ \\
\hline PT, INR & $1(0.8-2.1)$ & $1.1(0.9-2.6)$ & $<0.001$ \\
\hline aPTT, sec & $35(27-75 \cdot 3)$ & $37.2(27.4-74.8)$ & $<0.001$ \\
\hline Ferritin, ng/mL & $78.24(3.91-1,223)$ & $292.15(4.65-71,627)$ & $<0.001$ \\
\hline LDH, IU/L & $370(242-1,231)$ & $447(207-33,241)$ & $<0.001$ \\
\hline BUN, mg/dL & $15(5.2-71.5)$ & $17.1(2.2-129.5)$ & 0.001 \\
\hline $\mathrm{Cr}, \mathrm{mg} / \mathrm{dL}$ & $0.67(0.26-8.13)$ & $0.7(0.11-10.04)$ & 0.381 \\
\hline $\mathrm{CRP}, \mathrm{mg} / \mathrm{L}$ & $1.43(0-146.98)$ & $7.98(0-324.83)$ & $<0.001$ \\
\hline
\end{tabular}

Values are presented as median (range).

ITP, immune thrombocytopenic purpura; IPF, immature platelet fraction; BM, bone marrow; PLT, platelet; Hb, hemoglobin; WBC, white blood cell; PT, prothrombin time; INR, international normalized ratio; aPTT, activated partial thromboplastin time; LDH, lactate dehydrogenase; BUN, blood urea nitrogen; Cr, creatinine; CRP, C-reactive protein. 


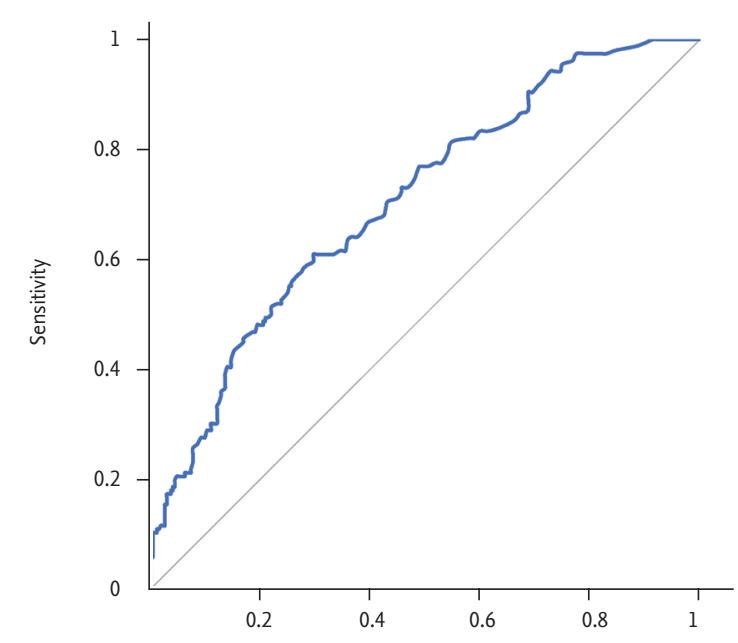

A

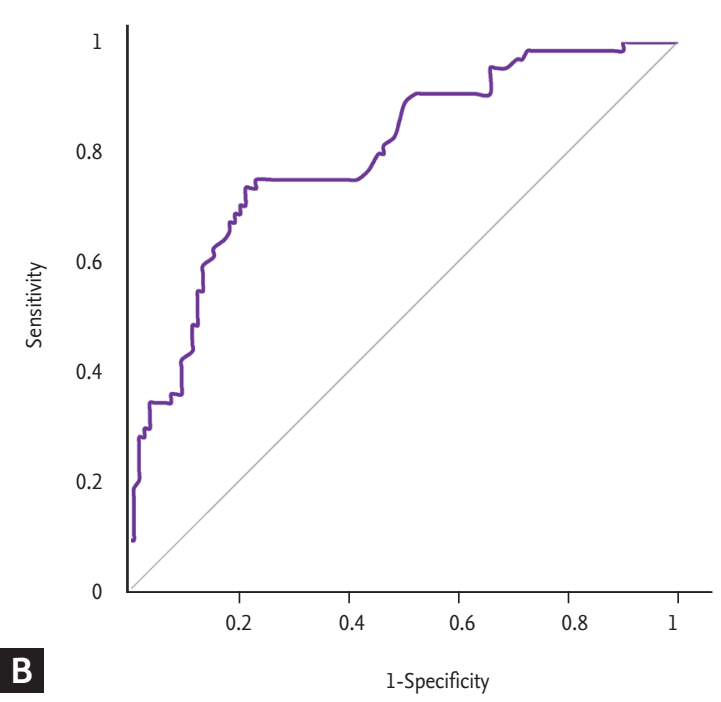

Figure 1. (A) Receiver-operating characteristic (ROC) curve of immature platelet fraction (IPF) in the immune thrombocytopenic purpura (ITP) group and non-ITP group. The optimal cut-off value of IPF (\%) for differentiating ITP was $7.0 \%$ with a sensitivity of $61 \%$ and specificity of $70 \%$. (B) ROC curve of IPF in the ITP group and non-ITP subgroups of patients subjected to a bone marrow examination. The optimal cut-off value was $7.8 \%$, with a sensitivity of $75 \%$ and a specificity of $76.7 \%$. The median IPF was higher in this subgroup and the sensitivity and specificity of the cut-off value were also higher than the former group.

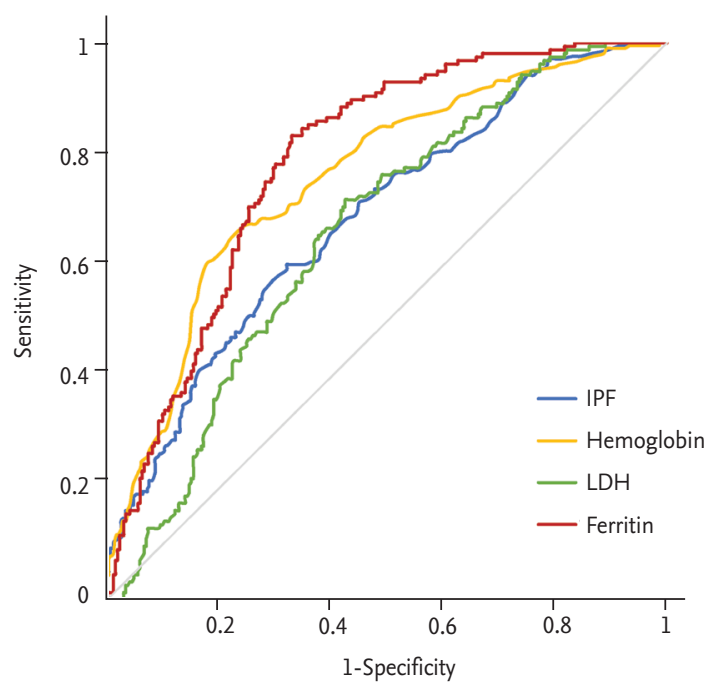

Figure 2. Receiver-operating characteristic (ROC) curve of immature platelet fraction (IPF), hemoglobin, lactate dehydrogenase (LDH), and ferritin. We determined an optimal cut-off value using ROC curve analysis. The optimal cut-off values were $7 \%, 12 \mathrm{~g} / \mathrm{dL}, 420 \mathrm{IU} / \mathrm{L}$, and $177 \mathrm{mg} / \mathrm{mL}$, respectively $(p<0.001, p<0.001, p=0.047$, and $p=0.001$, respectively $)$.

not be used to diagnose ITP, we also evaluated other laboratory variables via logistic regression analysis. Hemoglobin, $\mathrm{LDH}$, and ferritin showed statistical significance with $p<0.001, p=0.011$, and $p<0.001$, respectively. The optimal cut-off value of hemoglobin for distinguishing between ITP and non-ITP was $12 \mathrm{~g} / \mathrm{dL}$ with a sensitivity of $60 \%$ and specificity of $81.3 \%$. The optimal cut-off value of LDH was $420 \mathrm{IU} / \mathrm{L}$ (upper limit of normal [ULN]) with sensitivity $57.9 \%$, specificity $70.5 \%$ and the cut-off value of ferritin was $177 \mathrm{mg} / \mathrm{mL}$ with sensitivity 67\%, specificity $83.3 \%$ (Fig. 2).

\section{Derivation of predictive model}

These four independent variables which were identified through the logistic regression analysis comprised a simple diagnostic predictive scoring model (Table 3). To adequately reflect sensitivity and specificity, we divided patients into three groups according to IPF; IPF $<7, \geq 7$ and $<10, \geq 10$. Our model gave point to each of variables; 1 to high hemoglobin level (>12 g/dL), low ferritin level $(\leq 177 \mathrm{ng} / \mathrm{mL})$, normal LDH level $(\leq \mathrm{ULN})$ and $\mathrm{IPF} \geq 7$ and $<10$; and 2 to IPF $\geq 10$. The final score was obtained by summing the points (Table 4). The scoring model underwent validation. In the internal validation cohort consisting of patients who underwent bone marrow examination, a total of 166 patients had all variables of the scoring system. Sixty-four patients were diagnosed with ITP and 102 patients were diagnosed with 
Table 3. Results of logistic regression analysis for ITP diagnosis

\begin{tabular}{|c|c|c|c|}
\hline \multirow{2}{*}{ Laboratory variable } & \multirow{2}{*}{$\begin{array}{c}\text { Univariate analysis } \\
\text { p value }\end{array}$} & \multicolumn{2}{|c|}{ Multivariate analysis } \\
\hline & & $\mathrm{HR}(95 \% \mathrm{CI})$ & $p$ value \\
\hline IPF, \% & $<0.001$ & $1.198(1.125-1.277)$ & $<0.001$ \\
\hline Hemoglobin, g/dL & $<0.001$ & $1.398(1.176-1.662)$ & $<0.001$ \\
\hline LDH, IU/L & $<0.001$ & $0.998(0.996-1.000)$ & 0.047 \\
\hline Ferritin, ng/mL & $<0.001$ & $0.997(0.995-0.999)$ & 0.001 \\
\hline Reticulocyte, \% & 0.329 & & \\
\hline WBC, $\times 10^{9} / \mathrm{L}$ & 0.685 & & \\
\hline Protein, g/dL & 0.299 & & \\
\hline Albumin, g/dL & $<0.001$ & & \\
\hline Bilirubin, mg/dL & $<0.001$ & & \\
\hline PT, INR & $<0.001$ & & \\
\hline aPTT, sec & $<0.001$ & & \\
\hline BUN, mg/dL & $<0.001$ & & \\
\hline $\mathrm{Cr}, \mathrm{mg} / \mathrm{dL}$ & 0.055 & & \\
\hline CRP, mg/L & $<0.001$ & & \\
\hline
\end{tabular}

ITP, immune thrombocytopenic purpura; HR, hazard ratio; CI, confidence interval; IPF, immature platelet fraction; LDH, lactate dehydrogenase; WBC, white blood cell; PT, prothrombin time; INR, international normalized ratio; aPTT, activated partial thromboplastin time; BUN, blood urea nitrogen; Cr, creatinine; CRP, C-reactive protein.

Table 4. Validation of diagnostic predictive scoring model

\begin{tabular}{|c|c|c|}
\hline \multirow{2}{*}{ Variable } & \multicolumn{2}{|c|}{ No. of ITP patients } \\
\hline & Internal validationcohort $(n=166)$ & External validation cohort $(\mathrm{n}=171)$ \\
\hline \multicolumn{3}{|l|}{ Total score } \\
\hline o & $0 / 28(0)$ & $0 / 40(0)$ \\
\hline 1 & $4 / 31(12.9)$ & $\mathrm{o} / 50(0)$ \\
\hline 2 & $5 / 40(12.5)$ & $3 / 42(7.1)$ \\
\hline 3 & $26 / 37(70.3)$ & $16 / 25(64)$ \\
\hline 4 & $23 / 23(100)$ & $7 / 11(63.6)$ \\
\hline 5 & $6 / 7(85.7)$ & $3 / 3(100)$ \\
\hline \multicolumn{3}{|l|}{ Diagnosis } \\
\hline Non-ITP (o-2) & $9 / 99(9)$ & $3 / 132(2.3)$ \\
\hline $\operatorname{ITP}(3-5)$ & $55 / 67(82.1)$ & $26 / 39(66.7)$ \\
\hline
\end{tabular}

Values are presented as number (\%). Data are total number of patients with ITP/total number of patients with that score. Scoring model: 1 point to high hemoglobin $(>12 \mathrm{~g} / \mathrm{dL})$, low ferritin $(\leq 177 \mathrm{ng} / \mathrm{mL})$, normal lactate dehydrogenase $(\leq$ upper limit of normal) and immature platelet fraction (IPF) $\geq 7$ and $<10,2$ point to IPF $\geq 10$.

ITP, immune thrombocytopenic purpura.

non-ITP diseases. In this cohort, none of the 64 patients with ITP were assigned a score of 0 ; 55 patients (85.9\%) were assigned a score of $3-5$. We defined that ITP could be highly predicted in patients with more than 3 points.
Accuracy, precision and recall were $87 \%, 82 \%$, and $86 \%$, respectively. We also applied this scoring model to the external validation cohort. The external validation cohort included patients with thrombocytopenia (platelet 
count $<100 \times 10^{9} / \mathrm{L}$ ) of various causes who presented to Korea University Guro Hospital from January 2018 to December 2018 or Korea University Anam Hospital from January 2017 to December 2018. A total of 171 patients who underwent bone marrow examination had all variables and could be scored. Twenty-nine patients were diagnosed with ITP and 142 patients were diagnosed as non-ITP. None of 29 patients with ITP were assigned a score of o to $1 ; 26$ patients (89.7\%) were assigned a score of 3 to 5 . We also found that ITP could be highly predicted in patients with more than 3 points. Accuracy, precision and recall were $91 \%, 67 \%$, and $90 \%$, respectively.

\section{DISCUSSION}

The purpose of this study is to find novel markers to diagnose ITP more easily and accurately, because there is no gold standard method for diagnosis of IT'P so far. In this study, we investigated the usefulness of IPF (\%) for the differential diagnosis of ITP and found that IPF (\%) is significantly higher in ITP patients than in the nonITP group. The role of IPF has been reported in several studies. Briggs et al. [15] showed that IPF is higher in ITP and thrombotic thrombocytopenic purpura, which are associated with excessive peripheral consumption of platelets than in the group undergoing cytotoxic chemotherapy. Jung et al. [17] confirmed that the median $\operatorname{IPF}(\%)$ of ITP patients is $7.7 \%$, that of AA patients is $3.5 \%$ and the cut-off value of IPF differentiating ITP from AA was 7.3\%. Naz et al. [21] compared the IPF (\%) of ITP patients with other thrombocytopenic patients and normal healthy adults and observed that IPF (\%) was higher in the ITP group than in the non-ITP group. These studies presented a similar conclusion that IPF (\%) might be a diagnostic parameter of patients with ITP; however, these have several limitations as follows. (1) They mainly compared the IPF (\%) of patients with ITP and that of normal control groups without thrombocytopenia, and the size of the control groups with thrombocytopenia were too small. (2) There were no studies comparing the IPF (\%) in patients with and without bone marrow examination. Since most studies did not use bone marrow examination for the diagnosis of ITP, some patients might have been misdiagnosed as ITP. (3) The criterion for thrombocytopenia was platelet count less than $150 \times$ 10\%/L, which was inconsistent with the criteria from the International Working Group.

In this study, we included 568 patients with platelet count less than $10 \times 109 / \mathrm{L}$ for both the ITP and nonITP groups. Even though only patients with less than 100,000 platelets were included in the study, the study was conducted on a relatively large number of patients compared to previous studies. Bone marrow examinations were not performed in all patients, but we performed bone marrow examination in almost half of patients for diagnosis and clearly excluded the possibility of other diseases. In this way, since other cause of thrombocytopenia was excluded in a large number of patients with bone marrow examination, our study diagnosed ITP more accurately compared to previous studies. IPF could be expressed as an absolute count or a percentage. We used the IPF (\%) because the absolute value of immature platelets may also be low if the platelet count is low. The median IPF (\%) for patients with ITP was significantly higher compared to that of the non-IT'P group. Therefore, our result indicated that IPF (\%) could be applied as a useful parameter to distinguish the cause of thrombocytopenia, especially in the diagnosis of ITP. The optimal cut-off value to differentiate ITP from other causes of thrombocytopenia was 7.0\%, with a sensitivity of $61 \%$ and a specificity of $70 \%$. These are similar results to those of previous studies. Since ITP is diagnosed through the exclusion of other diseases, the initial diagnosis may not be accurate, especially, in patients with several underlying diseases or with a short follow-up period. Therefore, some patients who did not undergo bone marrow examination could be misdiagnosed with ITP and consequently misclassified into the ITP group in our study. To exclude the possibility of misclassification, only patients who underwent bone marrow examination were selected and included in the subgroup analysis. In this subgroup, the median $\operatorname{IPF}(\%)$ for patients with ITP was also higher than that of the non-IT'P group and the difference was even more pronounced. The optimal cut-off value for differentiating ITP from other causes of thrombocytopenia was $7.9 \%$, with a sensitivity of $75 \%$ and specificity of $76.7 \%$.

However, diagnosing ITP with IPF alone has some limitations. Although ITP is mainly attributed to immune-mediated platelet destruction, recently, inade- 
quate platelet production has also been suggested as a factor in the pathogenesis of ITP $[3,22]$. Also, ITP is considered a heterogeneous disease, as the etiology of thrombocytopenia differs among patients. IPF reflects only young platelet production, and cannot fully reflect the complicated pathogenesis of ITP. In addition, IPF may be similar in other diseases like liver disease with splenomegaly, according to the mechanism of thrombocytopenia. Furthermore, as platelets have a shorter lifespan relative to red blood cells, fluctuations and variations of IPF (\%) might be larger, depending on the time of the test. Therefore, we also measured other laboratory variables to overcome the limitation of IPF and improve the diagnostic accuracy of ITP. Hemoglobin, LDH, and ferritin showed statistical significance in the logistic regression analysis. Low hemoglobin, high $\mathrm{LDH}$, and high ferritin level were found to be associated with the non-ITP group. These may be attributable to the fact that the non-ITP group included patients with various bone marrow diseases. $\mathrm{LDH}$ and ferritin are also known as acute phase reactants, the levels of which can be increased by inflammation and tissue damage. Moreover, ferritin can be released from damaged hepatocytes and indirectly reflects liver damage. We developed a simple scoring model for the differential diagnosis of ITP based on these results and found it to assess the probability of ITP reliably. Because our predictive model consists of common laboratory variables that are frequently used in general clinical practice, we expect that it could be easily applied to patients in clinical practice instead of bone marrow examination.

In conclusion, IPF (\%) could be a useful parameter to distinguish ITP from thrombocytopenia of other causes. Though IPF (\%) alone is hardly a gold standard for the diagnosis of ITP, it could be more useful in combination with other laboratory variables. We developed a predictive scoring model for the differential diagnosis of ITP using IPF (\%), hemoglobin, LDH, and ferritin. External validation with a large cohort of patients is needed to determine the efficacy of this model.

\section{KEY MESSAGE}

1. Immature platelet fraction (IPF) could be a useful parameter to distinguish immune throm- bocytopenic purpura (ITP) from other causes of thrombocytopenia.

2. We developed the predictive scoring model using IPF, hemoglobin, lactate dehydrogenase, and ferritin. This model could predict ITP with high probability in patients with more than 3 points.

\section{Conflict of interest}

No potential conflict of interest relevant to this article was reported.

\section{REFERENCES}

1. Rodeghiero F, Stasi R, Gernsheimer T, et al. Standardization of terminology, definitions and outcome criteria in immune thrombocytopenic purpura of adults and children: report from an international working group. Blood 2009;113:2386-2393.

2. Portielje JE, Westendorp RG, Kluin-Nelemans HC, Brand A. Morbidity and mortality in adults with idiopathic thrombocytopenic purpura. Blood 2001;97:2549-2554.

3. Cooper N, Bussel J. The pathogenesis of immune thrombocytopaenic purpura. Br J Haematol 2006;133:364-374.

4. Cines DB, Bussel JB. How I treat idiopathic thrombocytopenic purpura (ITP). Blood 2005;106:2244-2251.

5. Provan D, Stasi R, Newland AC, et al. International consensus report on the investigation and management of primary immune thrombocytopenia. Blood 2010;115:168-186.

6. Jubelirer SJ, Harpold R. The role of the bone marrow examination in the diagnosis of immune thrombocytopenic purpura: case series and literature review. Clin Appl Thromb Hemost 2002;8:73-76.

7. Ault KA, Rinder HM, Mitchell J, Carmody MB, Vary CP, Hillman RS. The significance of platelets with increased RNA content (reticulated platelets): a measure of the rate of thrombopoiesis. Am J Clin Pathol 1992;98:637-646.

8. Ingram M, Coopersmith A. Reticulated platelets following acute blood loss. Br J Haematol 1969;17:225-229.

9. Karpatkin S. Human platelet senescence. Annu Rev Med 1972;23:101-128.

10. Chavda N, Mackie IJ, Porter JB, Harrison P, Patterson K, Machin SJ. Rapid flow cytometric quantitation of reticulated platelets in whole blood. Platelets 1996;7:189-194. 
11. Michelson AD. Flow cytometry: a clinical test of platelet function. Blood 1996;87:4925-4936.

12. Watanabe K, Takeuchi K, Kawai Y, Ikeda Y, Kubota F, Nakamoto H. Automated measurement of reticulated platelets in estimating thrombopoiesis. Eur J Haematol 1995:54:163-171.

13. Abe $\mathrm{Y}$, Wada $\mathrm{H}$, Tomatsu $\mathrm{H}$, et al. A simple technique to determine thrombopoiesis level using immature platelet fraction (IPF). Thromb Res 2006;118:463-469.

14. Briggs C, Harrison P, Grant D, Staves J, MacHin SJ. New quantitative parameters on a recently introduced automated blood cell counter: the XE 2100. Clin Lab Haematol 2000;22:345-350.

15. Briggs C, Kunka S, Hart D, Oguni S, Machin SJ. Assessment of an immature platelet fraction (IPF) in peripheral thrombocytopenia. Br J Haematol 2004;126:93-99.

16. Ferreira FLB, Colella MP, Medina SS, et al. Evaluation of the immature platelet fraction contribute to the differential diagnosis of hereditary, immune and other acquired thrombocytopenias. Sci Rep 2017;7:3355.

17. Jung H, Jeon HK, Kim HJ, Kim SH. Immature platelet fraction: establishment of a reference interval and diagnostic measure for thrombocytopenia. Korean J Lab Med 2010;30:451-459.

18. Ko YJ, Kim H, Hur M, et al. Establishment of reference interval for immature platelet fraction. Int J Lab Hematol 2013;35:528-533.

19. Nomura T, Kubota Y, Kitanaka A, et al. Immature platelet fraction measurement in patients with chronic liver disease: a convenient marker for evaluating cirrhotic change. Int J Lab Hematol 2010;32:299-306.

20. Pons I, Monteagudo M, Lucchetti G, et al. Correlation between immature platelet fraction and reticulated platelets. Usefulness in the etiology diagnosis of thrombocytopenia. Eur J Haematol 2010;85:158-163.

21. Naz A, Mukry SN, Shaikh MR, Bukhari AR, Shamsi TS. Importance of immature platelet fraction as predictor of immune thrombocytopenic purpura. Pak J Med Sci 2016;32:575-579.

22. Yazdanbakhsh K, Zhong H, Bao W. Immune dysregulation in immune thrombocytopenia. Semin Hematol 2013;50 Suppl 1:S63-S67. 